\title{
Subcutaneous Emphysema Complicating Convalescent Stage of Measles in a Malnourished Indigent Child: A Case Report from North-western Nigeria.
}

\author{
Aliyu Mamman Na'uzo (D NAUZOMAN@YAHOO.COM ) \\ Federal Medical Centre, Birnin Kebbi https://orcid.org/0000-0002-0048-2228 \\ Usman Abiola Sanni \\ Department of Paediatrics,Federal Medical Centre,Birnin Kebbi \\ Taslim Olatunde Lawal \\ Department of Pediatrics, Federal Medical Centre, Brinin Kebbi
}

\section{Tawakaltu Lilly Musa}

Department of Paediatrics, Federal Medical Centre, Birnin Kebbi

Ojumo Opeyemi Gabriel

Department of Paediatrics, Federal Medical Centre, Birnin Kebbi

\section{Zainab Abdullahi}

Department of Community Health, Usmanu Danfodiyo University Teaching Hospital

\section{Muhammad Ibrahim Habib}

Department of Plastic and Reconstructive Surgery, Federal Medical Centre, Birnin Kebbi

\section{Case Report}

Keywords: Measles, Subcutaneous emphysema, Indigent child, Malnutrition, Nigeria Word count: 194

Posted Date: September 7th, 2021

DOl: https://doi.org/10.21203/rs.3.rs-845700/v1

License: (c) (i) This work is licensed under a Creative Commons Attribution 4.0 International License. Read Full License 


\section{Abstract}

Background: Measles is a highly infectious vaccine preventable viral disease that runs a devastating course in developing countries due to its association with malnutrition and poor immunization coverage. Among the complications of measles, pneumonia accounts for most measles-associated morbidity and mortality. However, subcutaneous emphysema is a rare complication of measles that can be challenging to manage and may portend poor outcome if untreated.

Case presentation: We present a case of a $2 y \mathrm{r}$ old un-immunized rural dweller with massive subcutaneous emphysema comorbid with malnutrition complicating convalescent stage of measles. The child failed to improve with conservative management but responded to closed thoracostomy tube drainage (CTTD) through an underwater seal bottle with intermittent negative pressure wound therapy (NPWT). The child spent 47days on admission during which the treatment was supported by faith-based organization and social welfare unit of the hospital.

\section{Conclusion}

Subcutaneous emphysema is a rare complication of measles infection that can be challenging to manage especially when comorbid with malnutrition in an indigent child. Multi-disciplinary team approach and the use of CTTD with NPWT is an effective management measure which can shorten the duration of hospital stay.

\section{Background}

Measles is a highly infectious, vaccine preventable disease of major public health concern worldwide especially in the developing countries [1]. Despite the availability of safe and effective vaccine, in 2018 alone more than 140000 people, mostly children under the age of 5 died from measles [2].

Generally, Measles runs a more devastating course in children in developing countries because of high rate of undernutrition, overcrowding, and lack of access to care, with mortality as high as 1 to $15 \%$ [3]. The commonest severe respiratory complication of the disease is pneumonia which account for most measles related death [4]. However other rare respiratory complications such as mediastinal and subcutaneous emphysema (SE) do occur and are associated with poor outcome if untreated $[5,6]$.

We present a case of a 2 year old rural dweller with massive subcutaneous emphysema comorbid with malnutrition following measles. The patient benefited from closed thoracostomy tube drainage through an underwater seal bottle with intermittent negative pressure wound therapy and nutritional support with a favourable outcome after a prolonged hospital stay.

\section{Case Report}


The case is a 2 year old girl who was referred to our Emergency Paediatric Unit from a Secondary Health Care Centre with complaints of chest, neck and facial swellings 3days after she was managed and discharged for Measles from the referring hospital. She did not receive measles vaccination but had oral polio vaccine (OPV) during the supplementary immunization exercise. She had contact with other children who had measles infection among members of her household. She was not exclusively breastfed, and her complementary diet was poor. Her current diet was mainly carbohydrate for which she poorly tolerates with the onset of the illness. The parents are of low socio-economic class that engage in subsistence farming.

On examination, she was acute on chronically ill-looking, wasted and restless, mildly pale with hypopigmented hair, angular stomatitis and desquamating skin lesions on the trunk and upper limbs. She had massive peri-orbital, neck and chest wall swelling with subcutaneous crepitus extending down to the abdominal wall (Fig. 1). She was afebrile with no significant lymphadenopathy. Her weight was $54 \%$ of the expected with a Z-score of $<-4 S D$. Her Respiratory system examination shows a rate of $28 \mathrm{cpm}$ with resonant chest percussion and oxygen saturation (SPO2) of $99 \%$.

A provisional diagnosis of convalescent measles complicated by subcutaneous emphysema on background severe acute malnutrition was made.

Complete blood count shows WBC of $10.210^{3} /$ I with relative leukocytosis, Haematocrit of $28 \%$, and normal platelets counts. Retro-viral screening for HIV and reverse transcriptase PCR for COVID-19 were negative. Blood sample for Bact/Alert was positive and gram stain showed gram positive cocci. Subculture yielded moderate growth of streptococcus spp after 48 hours of incubation, sensitive to Erythromycin, Gentamicin and Linezolid acid but resistant to Vancomycin. Abdominal ultrasound scan revealed bilateral grade I renal parenchymal disease. Arterial blood gases show pH of 7.56, pCO2 of $51.1 \mathrm{mmHg}, \mathrm{pO} 2$ of $28 \mathrm{mmHg}$ and $\mathrm{HCO} 3$ of $46.3 \mathrm{mmol} / \mathrm{l}$ lactate of $2.02 \mathrm{mml} / \mathrm{l}$. E/U/Cr showed potassium of $2 \mathrm{mmol} / \mathrm{l}$ and ionized calcium of $1 \mathrm{mmol} / \mathrm{l}$. Chest X-ray reveled extensive soft tissue swelling in the neck and axillary region with areas of translucency. The lung fields were clear except for the perihilar opacities (Fig. 2).

Patient was reviewed by the Ophthalmologist, Otorhinolaryngologist, Paediatric Surgeon and Plastic Surgeon. She was initially placed on I.V Cefuroxime but changed to I.V Ampicillin/Sulbactam and Gentamicin, following blood culture result. She also had tabs Zinc, Vitamin A and nutritional support with therapeutic milk (F75 and F100). She was transfused with packed cells when the PCV dropped to $20 \%$. She Had corrections for both potassium and calcium derangements. Supplemental oxygen was also administered. Her condition initially improved within 48hrs after the change of antibiotic necessitating the consideration of gas producing organism as the cause of the emphysema. However, on the 16th day of admission her condition worsens. She had subcutaneous fenestrated $18 \mathrm{G}$ cannular insertion on the supraclavicular area, with the open end attached to a suction machine set at a pressure of $125 \mathrm{mmHg}$ to create a negative pressure. She improved initially for 5 days but the swelling reaccumulates afterwards. She had closed thoracostomy tube drainage inserted at the right triangle of safety and an underwater 
seal bottle with intermittent negative pressure drainage on the 28th day of admission with complete resolution of the subcutaneous emphysema within ten days. She had remarkable improvement with healed ulcers, acid - base and electrolyte corrected and gained weight. Over the forty-seven days on admission, most of the care was funded by the volunteered staff, social welfare unit and faith based NonGovernmental Organization since the parents are of low socioeconomic class. Figure 3 shows the time course for the patient while on admission.

\section{Discussion And Conclusions}

We report a case of spontaneous subcutaneous emphysema recalcitrant to treatment in a 2year old girl in convalescent stage of measles comorbid with malnutrition. Reported incidences of this rare complication varies from $0.59-1.5 \%$ [5]. The usual scenario is that with supportive treatment, the affected children rarely require invasive procedures for treatment; the emphysema undergo resolution within a short period of time with eventual recovery of the patient. Air leaks such as mediastinum and subcutaneous emphysema in patients with measles is postulated to result from rupture of alveoli because of increased intra-alveolar pressure in the bronchi vascular sheet that then travels through fascial planes to the mediastinum and subcutaneous tissues $[5,6]$. The hypothesis is that increased fragility of connective tissues due to both malnutrition and following the measles infection leads to the formation of bulla and pulmonary interstitial emphysema [5]. The index patient not only had subcutaneous emphysema complicating recent attack of measles but had an underlying malnutrition probably accounting for her prolonged hospital stay and recalcitrant course. Although emphysema complicating measles can occur at any age or stage of the disease, in a previous study, only a third of the cases presented during the eruptive stage. Therefore, the possibility of late presentation is of significance in clinical practice since relationship between the infection and subcutaneous emphysema may be missed in the absence of a rash, as seen in the present child. In most series majority of the cases cluster in children less than 5 years as it was with the index patient $[5,6]$. The presentation of massive subcutaneous emphysema comorbid with malnutrition in this case is similar to a case reported by $\mathrm{H}$. Ahmed et al from Nguru, north eastern Nigeria [7]. However, the index patient did not have pneumonia like the child reported by Ahmed et al.

Some differential diagnosis such as trauma, Asthma, and tuberculosis which are common in our environment and can mimic the presentation of the index patient were ruled out in the course of managing this patient. A study in Italy has reported that the occurrence of subcutaneous emphysema may be the first sign of tubercular disease in children [8]. Another study, although in adult patient, has documented the occurrence of pneumomediastinum and subcutaneous emphysema following SARSCOV-2 infection [9]. The index patient had both tuberculosis screening and COVID-19 test, which were all negative. The hilar lymphadenopathies on the chest X-ray may be the residual effect of previous infection or other pneumonic complication.

The treatment of subcutaneous emphysema is mainly supportive. The index patient, however, could not improve with supportive and minimally invasive management after two weeks on admission. This 
contrasted with the findings in Kano, Azare, and Somalia [5-7], and necessitated the decision to explore more invasive forms of treatment such as CTTD with negative pressure wound therapy.

One of the challenges we had in managing this patient was inability to perform a Chest CT because of financial constraint. Chest CT will be beneficial in confirming the diagnosis, assessment of any associated abnormalities such as the presence of a pleuro-cutaneous fistular and to exclude lifethreatening differential diagnoses [10].

The learning point here is that the coexistence of measles and malnutrition complicated by subcutaneous emphysema can be challenging to manage especially in an indigent child and may add to prolonged hospital stay. Multi-disciplinary team approach and the use of CTTD with NPWT is an effective management measure which can shorten the duration of hospital stay.

\section{Abbreviations}

CT: Computer tomography scan

CTTD: Closed thoracostomy tube drainage

HIV: Human immunodeficiency virus

NPWT: Negative pressure wound therapy

OPV: Oral polio vaccine

PCV: Pack cell volume

PCR: Polymerase chain reaction

SE: Subcutaneous emphysema

\section{Declarations}

\section{Ethical approval and consent to participate}

Written informed consent for publication was obtained from the parent and the Research and Ethics Committee of the institution (Reference number FMC/BK/HP/045/P/517/VOL.111)

\section{Consent for publication}

The authors certify that they had obtained all appropriate patient consent forms. In the form, parental consent for images used and other clinical information to be reported in the journal was obtained. The parent understands that name and initials will not be published, and due efforts will be made to conceal identity, but anonymity cannot be guaranteed 
Availability of data material

All data relating to this study are presented within the manuscript. Other materials are available from the corresponding author upon reasonable request.

\section{Conflict of interest}

The authors declare no conflict of interest

\section{Funding}

This work was not supported by any funding

\section{Author's contribution}

AMN wrote the first draft of this case report. SAU, TOL, TLM, OOG, ZA and IMH, all made significant contributions to the first major revision of the manuscript, final manuscript revisions and approval of the final version.

\section{Acknowledgment}

We acknowledge all stake holders involved in the management of this patient

\section{References}

1. World Health Organization. Measles vaccines: WHO position paper, April 2017 - Recommendations. Vaccine [Internet]. Elsevier Ltd; 2019;37:219-22. Available from: https://doi.org/10.1016/j.vaccine.2017.07.066.

2. World Health Organisation. Measles [Internet]. [cited $2021 \mathrm{Jul} 3$ ]. Available from: https://www.who.int/news-room/fact-sheets/detail/measles.

3. Dossari A, Kamal. Clinical Practice: Clinical Practice. J Gastroenterol Hepatol. 2015;30:13-26.

4. Perry RT, Halsey NA. The clinical significance of measles: A review. J Infect Dis. 2004;189.

5. Moons $P$, Thallinger M. High incidence of subcutaneous emphysema in children in a somali refugee camp during measles outbreak. Pediatr Infect Dis J. 2014;33:96-8.

6. Aliyu I. Air leak syndrome complicating measles: report of two cases. Menoufia Med J. 2016;29:174.

7. Ahmed $\mathrm{H}$, Alhaji MA. IU. Extensive subcutaneous emphysema complicating measles in a malnourished child:A case report. Sahel Med J. 2009;12:35-7.

8. Esposito S, Giannini A, Biondetti P, Bonelli N, Nosotti M, Bosis S, et al. Subcutaneous emphysema as the first relevant clinical sign of complicated tubercular lymph node disease in a child. BMC Infect Dis. 2013;13:2-5.

9. Elhakim TS, Abdul HS, Pelaez Romero C, Rodriguez-Fuentes Y. Spontaneous pneumomediastinum, pneumothorax and subcutaneous emphysema in COVID-19 pneumonia: A rare case and literature 
review. BMJ Case Rep. 2020;13:1-7.

10. Benlamkaddem S, Berdai M, Labib S, Harandou M. A Case of Spontaneous Pneumomediastinum with Subcutaneous Emphysema in Children. Children. 2018. p. 22.

Figures

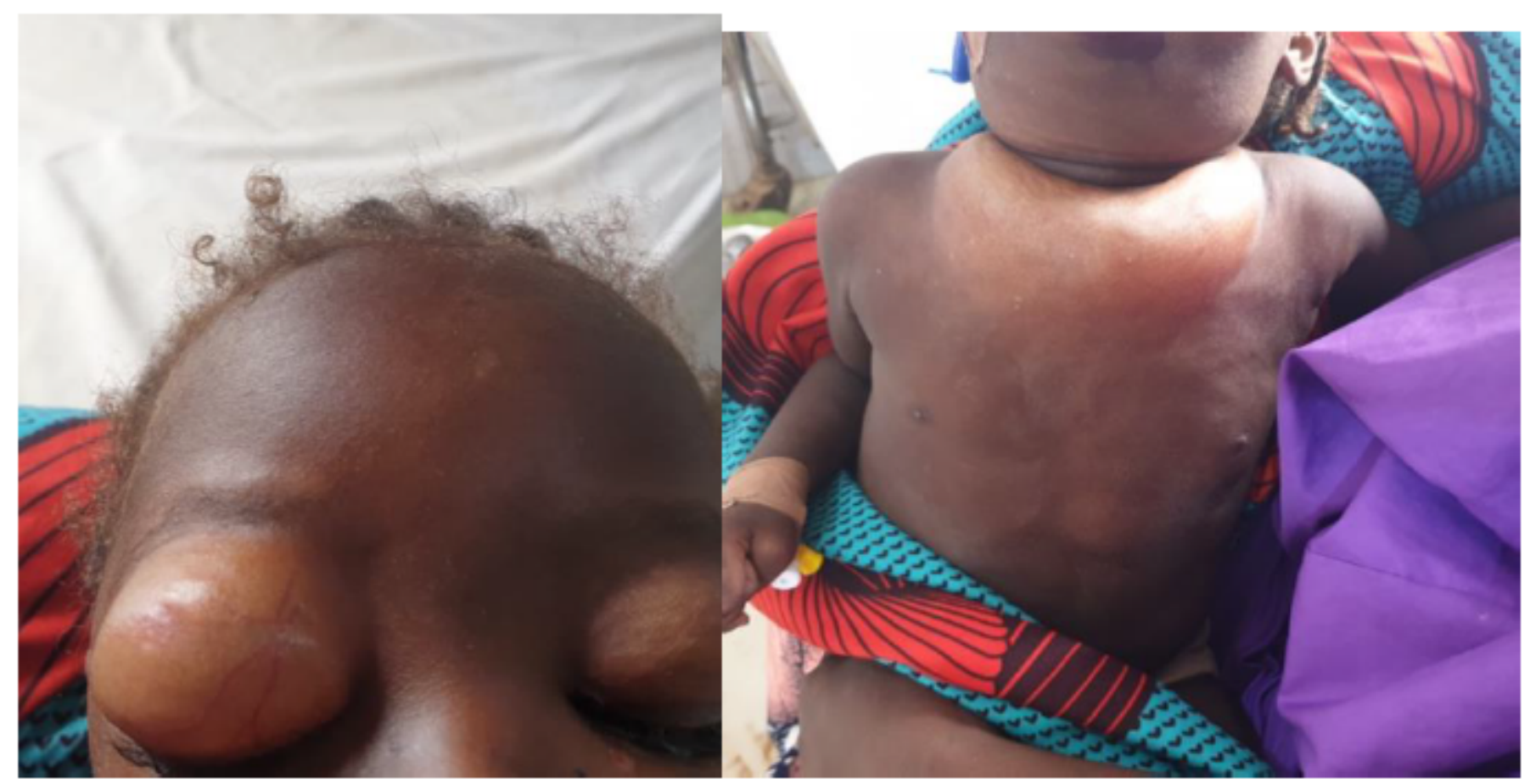

Figure 1

Massive subcutaneous emphysema involving the chest neck and eye lids
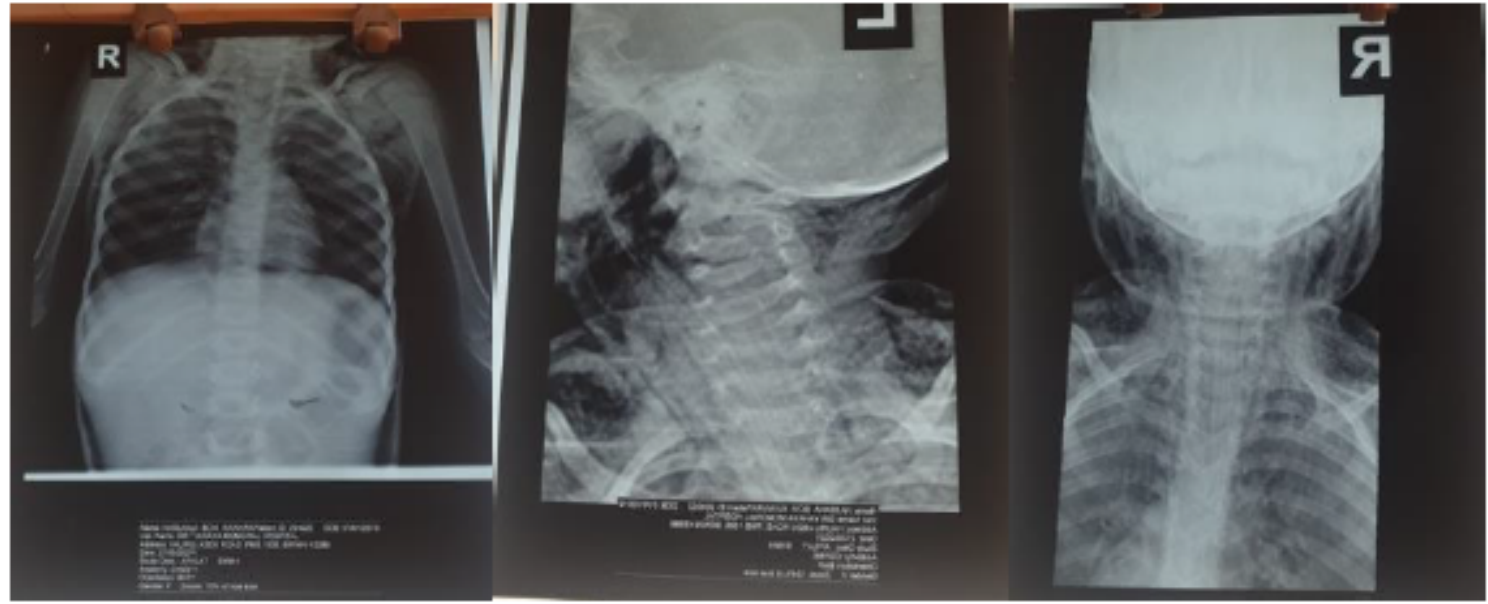

A

B

C

Figure 2 
(A) - Chest X-ray showing perihilar opacities. (B, C) - X-ray of the neck showing soft tissue swelling and extensive radiolucency within the subcutaneous tissue

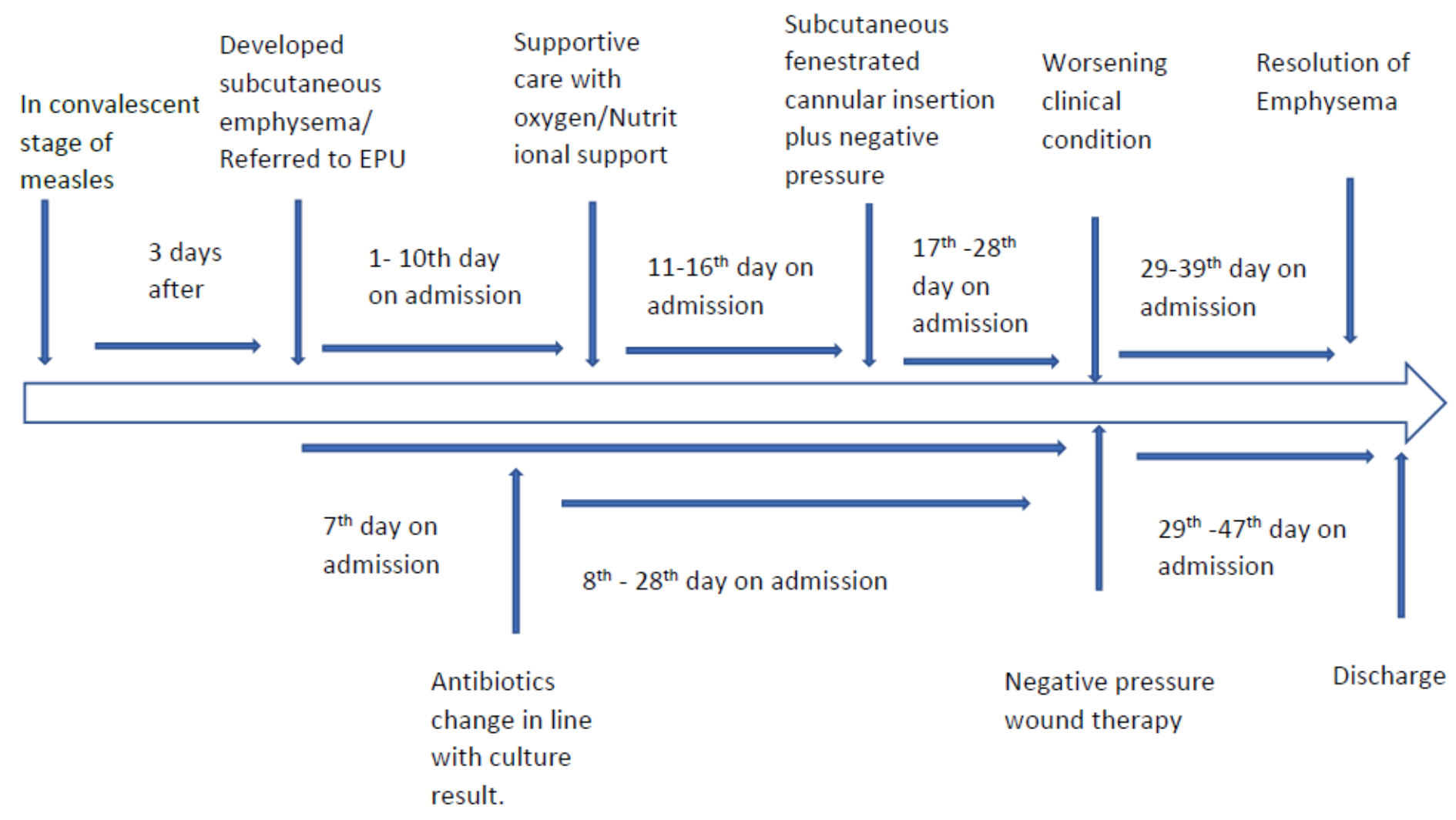

\section{Figure 3}

Time course of the patient while on admission 